\title{
AGGLOMERATION IN SOLID ROCKET PROPELLANTS: NOVEL EXPERIMENTAL AND MODELING METHODS
}

\author{
F. Maggi ${ }^{1}$, A. Bandera ${ }^{1}$, L. T. De Luca ${ }^{1}$, V. Thoorens ${ }^{2}$, \\ J. F. Trubert ${ }^{2}$, and T. L. Jackson ${ }^{3}$ \\ ${ }^{1}$ Politecnico di Milano \\ Aerospace Engineering Dept. \\ Via La Masa 34, Milan, Italy \\ ${ }^{2}$ ONERA, Departement d'Energétique Fondamentale et Appliquée \\ Chemin de la Hunière, Palaiseau, France \\ ${ }^{3}$ University of Illinois at Urbana-Champaign \\ CSE, Urbana, IL, USA
}

\begin{abstract}
Metal agglomeration is a key factor affecting the performance of metalized solid propellant rockets since many of the mechanisms that degrade specific impulse can be ascribed to metal powder aggregation and agglomeration. Condensed combustion products are generated at or near the burning surface of the propellant and then released in the gas phase where they are shaped by the core flow viscous forces and oxidized by the reactive environment. On this basis, detailed information about the size of agglomerates as they are generated from the propellant may improve the knowledge of the core flow and, thus, the prediction of its effects (namely, two-phase flow losses). This topic entails both modeling and experimental activities, and the aim of this work is to present some recent developments achieved at the Space Propulsion Laboratory in cooperation with other international institutions. The experimental part shows the application of high-speed and high-resolution imaging of propellant combustion for automated measurement of particle size exploited by means of an ad-hoc image processing tool. The modeling part demonstrates how heterogeneity can explain the agglomeration by means of a pocket model using spatial statistics and two- (2D) or threedimensional (3D) virtual propellants. A definition of a characteristic time that fits the agglomeration data for tested propellants is suggested, and a method for predicting the agglomerate size is given. Both activities are still a matter of research but the maturity level reached so far permits the application in some practical cases.
\end{abstract}

This is an Open Access article distributed under the terms of the Creative Commons Attribution-Noncommercial License 3.0, which permits unrestricted use, distribution, and reproduction in any noncommercial medium, provided the original work is properly cited. 


\section{NOMENCLATURE}

\section{Symbols}

$\begin{array}{ll}D_{\text {agg }} & \text { diameter of agglomerate, } \mu \mathrm{m} \\ d[4,3] & \text { mass-weighted mean diameter, } \mu \mathrm{m} \\ L^{*} & \text { characteristic length, } \mathrm{m} \\ p & \text { pressure, bar } \\ R & \text { radius in 3D agglomeration model, } \mu \mathrm{m} \\ r & \text { TPPF distance, } \mu \mathrm{m} \\ t_{\text {agg }}^{*} & \text { aggregation characteristic time, } \mathrm{s}\end{array}$

\section{Acronyms}

$\begin{array}{ll}\text { AP } & \text { Ammonium Perchlorate } \\ \text { CCP } & \text { Condensed Combustion Products } \\ \text { CT } & \text { Computed Tomography } \\ \text { HTPB } & \text { Hydroxyl Terminated PolyButadiene } \\ \text { MPI } & \text { Message Passing Interface } \\ \text { PDF } & \text { Probability Density Function } \\ \text { RGB } & \text { Red-Green-Blue color space } \\ \text { SOP } & \text { Smoke Oxide Particles } \\ \text { SRM } & \text { Solid Rocket Motor } \\ \text { TPPF } & \text { Two-Point Probability Function } \\ \text { UV } & \text { UltraViolet }\end{array}$

\section{INTRODUCTION}

Metal agglomeration is of primary importance for solid rocket propellant performance. The coalescence of the initial metal aggregates emerging at the burning surface and their subsequent inflammation eventually produces spherical agglomerates of molten material at or near the burning surface. These fused drops, made of metal and their oxides, enter the core flow of the rocket and move towards the nozzle, influencing the efficiency of the combustion chamber and of the nozzle. An AGARD workgroup (PEP WG-17) addressed the problem of evaluating actual performance of solid propellant rockets, and in their final report observed that about one third of the total specific impulse losses could be related to the presence of a two-phase flow inside the nozzle. Condensed mass fraction and size of the particles suspended in the nozzle flow were found to be relevant quantities for performance decrease, attributed to the thermal and velocity lag of the particles exhausted by the rocket $[1,2]$.

Detailed quantification of CCP (condensed combustion products) delivered from a propellant and injected inside a combustion chamber can be useful for the 
purpose of performance prediction. Information about particulate size can be retrieved through the collection of exhausted condensed products from the nozzle, correlated with rocket configurations and propellants as done by Hermsen [1]. Though this technique works for real rocket configurations and correlations between motor parameters and performance can be drawn, the original size distribution of particles leaving the propellant is still unknown by virtue of a continuous chemical and physical evolution whilst CCP travel within the hot and reactive core flow. This includes aluminum combustion and drop merging (convergent region) or breaking (divergent region).

Since it is not possible to infer any precise information about the original CCP size from the exhaust, specific measurements or modeling theories were proposed in the literature. Considerable work was carried on by Babuk et al. in the field of experimental collection techniques as well as in agglomeration modeling. Using a quenching plate or a liquid quenching pool, placed in the proximity of burning samples, CCP were promptly extinguished, accumulated, and made available to postcombustion analysis (measurement through laser granulometry, chemical analysis, or morphology [3]). Notwithstanding the validity of the technique for a complete analysis of the agglomerates, the method is intrusive and may alter some quality of the particles (shape, diameter, composition).

The experimental procedures to determine the size of CCP as close as possible to the burning surface can appeal to visualization techniques recently developed and to the ease of digital data handling. Using a high-speed and high-resolution videocamera coupled with an appropriate lighting system and a magnification apparatus, a population of particles can be captured and immobilized in a series of high-quality images, allowing nonintrusive measurements. The number of samples measured per propellant should be enough to ensure the reproducibility of results across different runs. Final outputs are the statistical descriptors of particle population.

Also, for modeling analysis and agglomerate size prediction, several pathways are explored at present, ranging from physical simulation to statistics. Solid propellants are heterogeneous materials, and statistics are capable of retrieving average descriptive features from the analysis of a bulk. Initial dataset for this kind of analysis consists of particle locations inside a sample of propellant, large enough to be representative. Real volumetric information can be obtained through X-ray microtomographic scans. Although, it is possible to generate a propellant numerical mockup through a technique of simulated particle packing, statistically equivalent to realistic conditions and conveniently fast, also for data processing.

The present paper works out a joint application of both experimental and modeling techniques for agglomerate characterization and prediction. Relying on high-speed and high-resolution visualizations, a consistent set of data was obtained from real propellants burning under known conditions. The CCP particle distributions were postprocessed and correlated to propellant microstructures 
through a statistical model. The same set of data was used for the tuning and validation of agglomeration models based on the statistical interpretation of propellant heterogeneous microstructures.

\section{AGGLOMERATION}

Combustion of aluminized composite propellants generates CCP of different sizes, covering a range of particles from submicrometric up to hundreds of microns in diameter. In general, the literature refers to two distinct categories of CCP, depending on size and source. Micronsized CCP derive from the agglomeration, partial combustion and final release of heated aluminum powders that reside on a burning surface for the time needed to complete the process, visible in Fig. 1. The final size of agglomerates ranges from tens to hundreds of microns, depending on residence times, formulation, burning rate, and combustion pressure. Agglomerates are burning particles of spherical shape, mainly constituted of molten Aluminum $(\mathrm{Al})$, a cap of Alumina $\left(\mathrm{Al}_{2} \mathrm{O}_{3}\right)$, and elements derived from the partial pyrolysis of binder. Leaving the burning surface, combustion of agglomerates progresses in the core flow of the rocket. Submicrometric CCP, referred to as smoke oxide particles (SOP), mainly come from the combustion of aluminum particles and agglomerates sustained by species diffusion around the metal drop [4]. Incomplete combustion of metal agglomerates may be promoted by a limited residence time in the combustion chamber, a small characteristic length $L^{*}$, low burning rate, or low combustion temperature, thus loosing the corresponding fraction of combustion enthalpy. In view of it, using the Beckstead's model in order to evaluate aluminum combustion time, it is possible to assume that rocket boosters used to equip large launch vehicles (e. g., Ariane 5 SRMs or VEGA P80) usually allow complete oxidation of metal fuel [5].

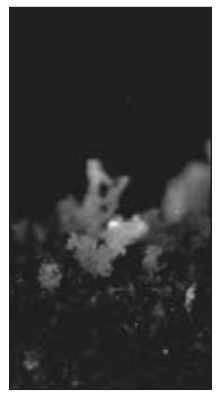

$0 \mathrm{~ms}$

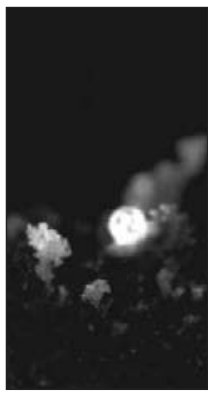

$18 \mathrm{~ms}$

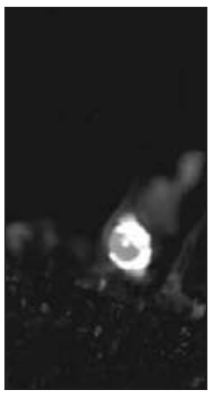

$33 \mathrm{~ms}$

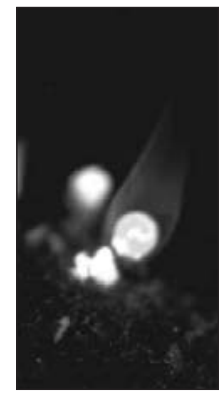

$65 \mathrm{~ms}$

Figure 1 Agglomeration sequence in a AP/HTPB/Al propellant burning at 750 mbar (courtesy of G. Colombo, SPLab, Politecnico di Milano, Italy) 
Within the class of standard composite rocket propellants, the agglomeration process takes place according to mechanisms mainly controlled by the coarse grains of ammonium perchlorate (AP) which draw a $3 \mathrm{D}$ random microstructure inside the bulk of a propellant. Bounded by the coarse AP particles, fuel-rich regions (called pockets) containing binder, aluminum, and fine oxidizer favor the generation of agglomerates. These regions are connected to each other by small strips of binder and aluminum powder (interpocket bridges). Trapped in this structure, aluminum particles move on the burning surface where a fraction of metal powder coalesces into larger clots under a variety of entraining and adhering forces. Here, aggregates are generated. Once exposed to the flame zone, subsequent inflammation occurs for both single and grouped particles, thus transforming aggregates into agglomerates. The final size of the agglomerates is strongly influenced by the residence time of the metal powders on the surface. In case of propellants containing micrometric aluminum powders, fast- and slowburning conditions should generate different scenarios as described in $[3,4,6]$. In the former conditions, the reduced residence time results in the so-called subpocket agglomeration that generates more agglomerates from one original pocket. In the latter conditions, the increased residence time can promote the merging of many agglomerates before detachment from the surface.

\section{EXPERIMENTAL TECHNIQUE}

Relevant to agglomeration, the collection of a reliable set of data is fundamental in the definition, validation, and tuning of a model. A consistent dataset should rely on consolidated measurement techniques, looking over a population of representative samples large enough to grant reproducible sets of data. According to previous experiences, 100 samples represent a minimum quantity. In order to connect the microstructure of a propellant to agglomerates generated, experimental analysis should focus on CCP just after the release from the burning surface. This rapid analysis should consider particles before any sort of modification due to the interaction with the core flow (of both chemical and fluid dynamic nature). For this reason, an optical technique for CCP measurement was implemented and optimized.

The agglomeration process was explored experimentally by measuring the size of CCP as a function of formulation and pressure over a range, for the time being, between 5 and 25 bar, in a closed vessel of about 21 of volume filled with nitrogen gas. The upper pressure bound is a constraint imposed by the measurement technique. In fact, both propellant burning rate and mass flow rate of combustion products grow with pressure; agglomerates are smaller and their speed increases accordingly. Under these conditions, higher shutter speed is required but lighting conditions worsen. 


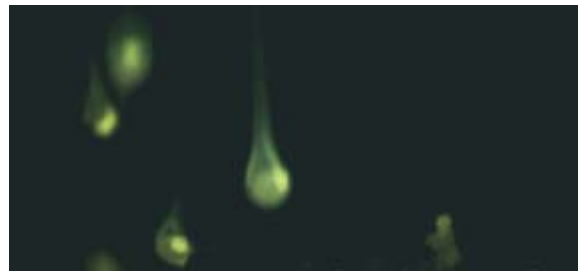

(a)

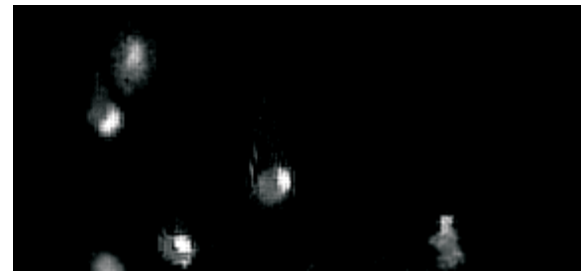

(b)

Figure 2 True colors $(a)$ and red layer minus blue layer (b). (Refer color plate, p. 86.)

Visualization of the combustion process was performed by means of a highspeed camera and a long-range microscope focused at least $5 \mathrm{~mm}$ below the ignition section. The experimentalist measured the agglomerates one-by-one from a selection of frames containing well defined items. Despite the method was operator-dependent, the reproducibility of measurements was tested by comparing the analyses performed by different people over the same sets of visualizations.

An attempt to create an automatic algorithm for agglomerates detection and size measurement has been made and is presented in this study. The objectives of this image processing were fast measurements and reliable statistics. The automatic particle sizing application relied on ImageJ, a free image processing software working under JAVA ${ }^{\mathrm{TM}}$ platform, supported by the National Institute of Health (NIH) of the United States [7]. The software may be easily extended by writing new algorithms with two different programming languages: Script or JAVA. The former uses ImageJ integrated functions whereas JAVA allows for low level programming. For this work, the programming language Script, with integrated functions and algorithms, was sufficient to meet the objectives. In conjunction with the algorithm development, optimal parameters of video acquisition were chosen to feed the software with images of better quality than in the past.

The software searches for agglomerates within a user-defined area of interest located just above the burning surface. The main challenge with this kind of image processing consists of removing with precision the tail that surrounds the agglomerates during their path inside the gas phase. An efficient method takes advantage of the characteristics of emitted light since aluminum combustion emits in the near UV spectrum. The tail is erased through a subtraction of the blue layer from the red one in the color space which gives the results shown in Fig. 2.

Being the ratio of "red/blue" light intensity higher in the particle than in the tail, the operation of subtraction clears the particles almost completely. After 


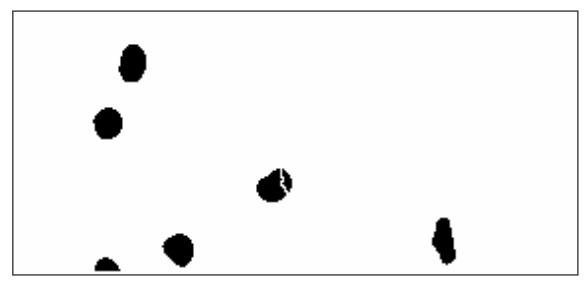

(a)

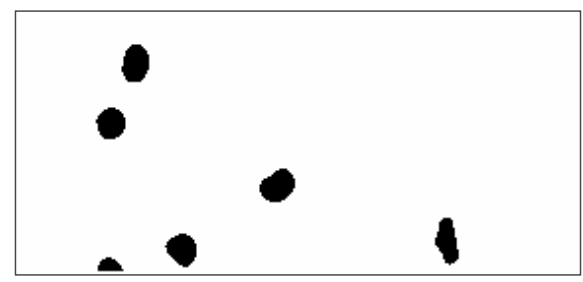

(b)

Figure 3 Application of watershed algorithm: (a) segmented picture with watershed; and $(b)$ merged areas from mathematical morphology

this preparation, a watershed algorithm is applied to the picture (Fig. $3 a$ ) to identify the luminous clusters [8]. Sometimes, after watershed, aluminum and alumina may not be in the same black area (an example is visible in the center on Fig. $3 b$ ) misleading the software to treat as two different particles. The correction of the problem can be carried through by mathematical morphology [9], used to merge these two areas. The result is the black and white picture reported in Fig. $3 b$.

Finally, a circularity test on black areas gives the possibility to remove small areas (numerical noise), areas not assimilated to a circle (flames or tails not removed),

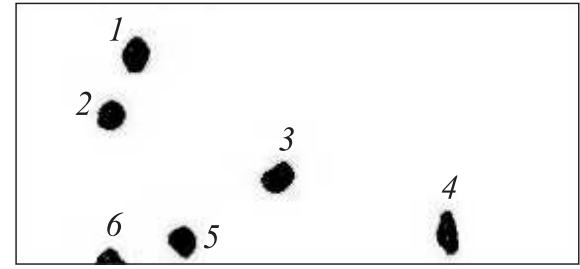

Figure 4 Detected particles

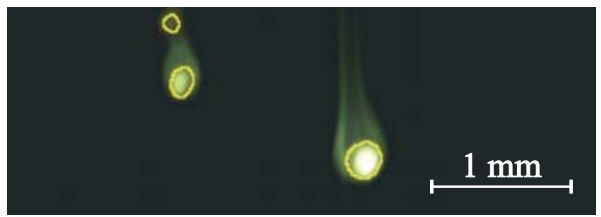

Figure 5 Detection results. (Refer color plate, p. 87.) and areas on the picture edge (wrong measures).

At this point, the ImageJ tools measures height, width, and perimeter of aluminum agglomerates detected in Fig. 4. Height and perimeter are not always reliable because of particle speed or the tail leftovers that may generate the distortion of particle shape along the vertical direction; so, "horizontal diameter" is considered to be a more representative characteristic dimension.

For comparison purposes, Fig. 5 shows the results of contour detection done with this algorithm overlapped to the original RGB image.

It should be noted that this kind of image processing is based on color visualization, with the detection of aluminum particles performed with RGB splitting and subtraction. Without a color camera, the algorithm does not work. 


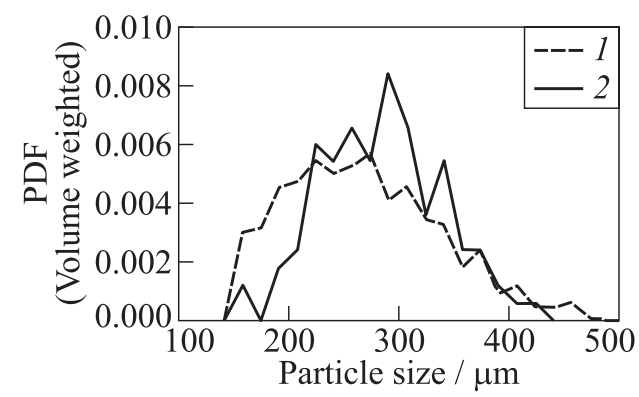

Figure 6 Statistics of automated (1, 999 values) and hand-made (2, 100 values) experimental measurements
As expected, the automated procedure could perform a higher number of measurements in less time with respect to the fully manual method. For example, the automated routine takes $10 \mathrm{~min}$ for 1000 values, whilst manual measurement takes several hours for a dataset of 100 values. Moreover, considerations about the output data quality and shape of PDFs (Fig. 6) suggest that the automated algorithm favors the capturing capability in the range of the small particles.

Figure 6 shows a comparison between manual and automated measurements. The similarity between the PDFs is encouraging since they share both the measured range of radii and the magnitude trend of the statistical descriptor, verifying that the automated procedure is consistent with the previous manual approach. The tests reveal also that automated image processing works correctly for pressures below 10 bar. At higher pressures, imaging quality decreases since particles travel at a higher speed. Moreover, a higher number of particles are present at the same time in the image and the algorithm encounters some problems of identification.

\section{INVESTIGATION ON MICROSTRUCTURE}

The connection between propellant microstructure and agglomerates was first identified in the 1960s by Povinelli and Rosenstein, who contrasted the influence of oxidizer size on collected agglomerates [10]. The heterogeneous nature of propellant was also addressed by several modeling approaches, establishing the idea of the microstructure dominated by pocket-like regions where agglomeration finds a privileged location to take place [11]. In his paper, Cohen also established some rules for the definition of final agglomerate size based on local flame temperature and encapsulation criteria, disregarding the random nature of the propellant microstructure. Microstructure and CCP were also investigated by Russian researchers through recent experimental as well as modeling works. In particular, Babuk et al. modeled the generation of agglomerates through a detailed physical description of the process but also looked at the aluminum pocket through a statistical approach [12]. Babuk et al. also made experimental works 


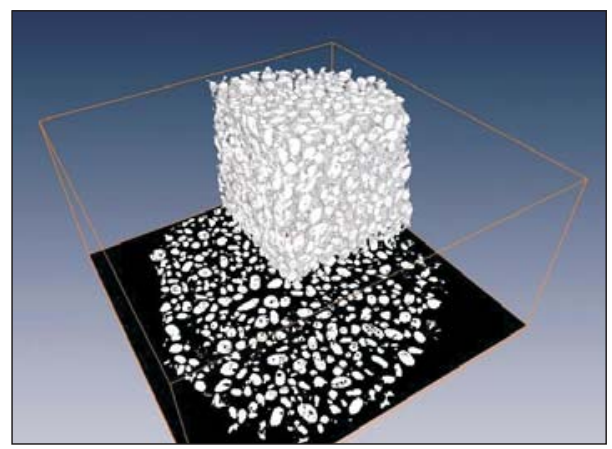

(a)

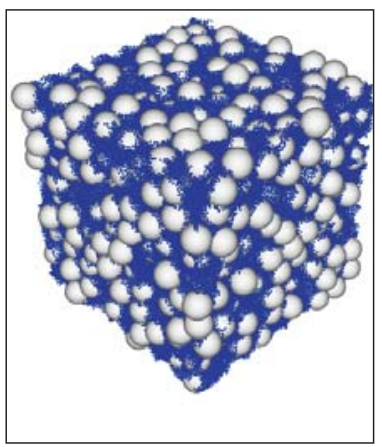

(b)

Figure 7 Datasets for the investigation of propellant inner structure [14]: (a) 3D Micro-CT scan of a nonaluminized propellant (cylinder diameter of $5 \mathrm{~mm}$ ); and (b) 3D propellant pack (grey spheres of $400 \mu \mathrm{m}$ and blue spheres of $30 \mu \mathrm{m}$ ). (Refer color plate, p. 89.)

of CCP collection and measurement of propellants embedding oxidizers of varying sizes and providing agglomeration data as a function of the propellant microstructure [3].

The statistical approach to heterogeneity was supported also by the research activities of Rashkovskii, who explored the joint use of statistics and packing codes for the generation of propellant numerical mockups used for agglomeration modeling [13]. Most of the theoretical studies on heterogeneity were and are grounded on numerical reconstruction of the microstructure by means of specific codes, though experimental methods of X-ray microtomography are applicable as well (Fig. 7) [15, 16]. Actually, the statistical analysis of CT datasets requires the identification of the ingredients inside the scanned volume by taking advantage of different absorption behavior of X-rays. The use of packing codes overcomes these problems. Some novel theoretical works can be found in the literature by Bandera et al. [17], Maggi et al. [18] as well as by Gallier et al. [19]. All of these references include experimental data used for the validation of agglomeration models.

Heterogeneous propellant microstructure is investigated through the analysis of $2 \mathrm{D}$ discs or $3 \mathrm{D}$ spheres. Datasets are numerically generated by packing applications. The 2D sections come from a disk packer by Bandera [20] and are used to draw a connection among agglomeration data, burning rate features, and heterogeneity through a general fitting curve. A 3D packer developed at CSAR $[21,22]$ is used to produce volumetric information of simulated propellant packs, then used to explore the functionality of a novel agglomeration model capable of predicting detailed CCP size distribution. Comparison to experimental data is provided. 


\section{MICROSTRUCTURE AND AGGLOMERATION: CORRELATION FROM TWO-DIMENSIONAL STATISTICS}

The first proposed statistical approach to agglomeration analyzes the heterogeneity of solid propellants aiming at the definition of a characteristic size of the pocket structure generated by the coarse oxidizer fraction. In this perspective, the statistical descriptor known as "Two-Point Probability Function", can be applied to characterize the reciprocal position of AP with respect to binder phase (here, the binder comprises also $\mathrm{Al}$ and fine AP). The maximum of this function represents the highest probability of phase change [23]. On the one hand, this kind of analysis can be conducted on real propellant sections after adequate augmentation of contrast which permits facilitated detection of coarse AP particles, as shown in Fig. 8. On the other hand, numerically generated sections reported in Fig. 9 reproduce the same propellant of Fig. 8 and may be used for statistical analysis as well. Aforementioned propellants comprehend $55 \%$ of volume occupation of coarse AP cut.

The numerical propellant sections analyzed in this work have been created in a pure $2 \mathrm{D}$ framework. The generating procedure considers a hard sphere representing an AP crystal that is cut by several parallel planes, randomly distributed along the diameter, thus creating circular surfaces of different radii. The creation of $2 \mathrm{D}$ circular sections progresses till the ratio between the sum of all generated circles and the total area of the section-to-be is equal to the volumetric occupation of the particles inside the compound. In a similar manner, when bimodal oxidizer distributions are used, the volume occupied by any single phase must be reached by surfaces generated from the corresponding phase. Once the sections have been generated, they are randomly placed inside the domain, one

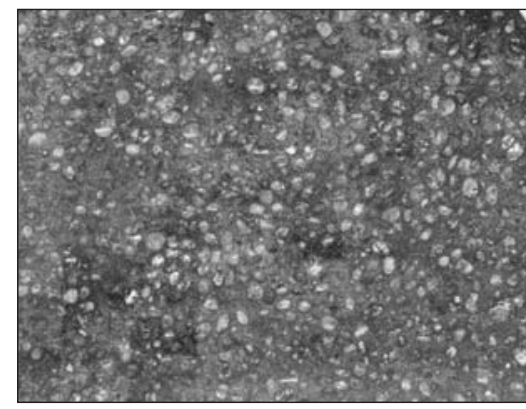

(a)

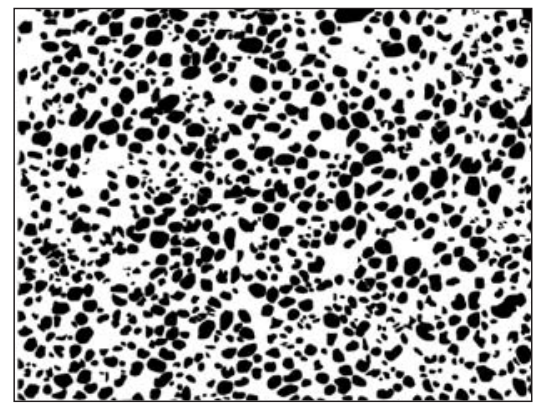

(b)

Figure 8 Detection of AP particles from a propellant section: (a) propellant section; and (b) AP particles detected 


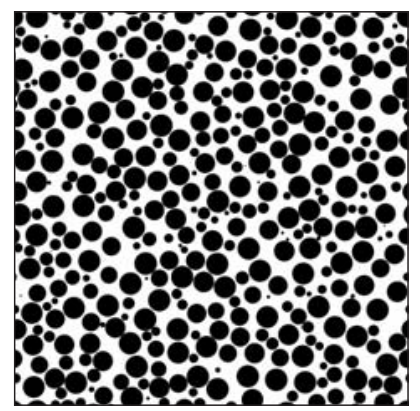

(a)

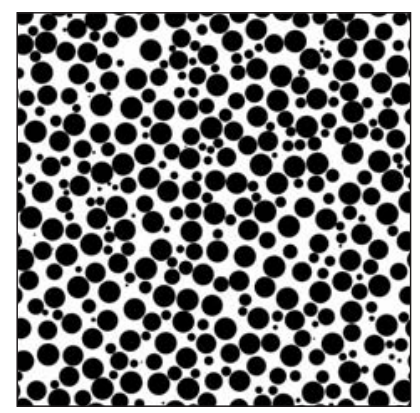

(b)

Figure 9 Two numerical sections generated by a packing code

by one, starting from the biggest disks. At each step of the procedure, particle overlapping is controlled before the acceptance of one new particle position. For the purpose of this work, the interest lies in the structure of the propellant located among the coarse oxidizer particles, since it is in this region that the population of aggregates/agglomerates undergoes its evolution. On this basis, the finer fraction of the oxidizer $(5-10 \mu \mathrm{m})$ is not included in the AP phase. As a consequence, only two phases are recognized in the created sections: AP phase (including large AP grains) and binder phase (including HTPB, fine AP, and metal).

The algorithm for the calculation of a TPPF is based on the construction of a circular sampling template with radial resolution of $1 \mu \mathrm{m}$, tangential resolution of $10^{\circ}$, and the condition of periodicity in order to follow the periodic boundary conditions of the simulated sections. Each statistical curve was formed by forty thousand random throws of the template. After testing the isotropy of the sections, the TPPF can be expressed as a function of the distance $r$ only.

Four different functions have been calculated in this work: AP-AP, BinderBinder, AP-Binder, and Binder-AP. The curves for AP-AP and Binder-Binder, for $r \rightarrow 0$, are equal to the volumetric occupation of the phases in the propellant compound. The Binder-AP and the AP-Binder represent the probability that phase is changed at a given distance from the center of the template and it is trivially zero for $r \rightarrow 0$. The maximum of this curve is the most important parameter. Keeping in mind the succession of regions filled with binder-metal and coarse oxidizer inside the propellant compound, the maximum of the curves can be identified as the maximum probability to step out from a binder region and move into an oxidizer particle, or vice versa. The location of this maximum, which will be referred to as the "characteristic size," is indicative of the size of the microstructures that are created inside the compound. Furthermore, it can be noted that the maximum of these curves is connected with the minimum of the homogeneous phase curves. 


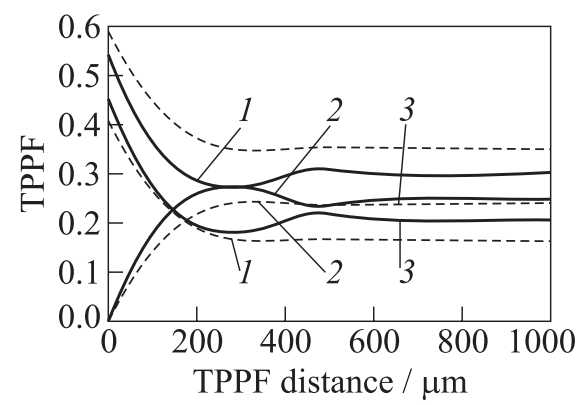

Figure 10 Two-Point Probability Functions of numerical (solid curves) and experimental (dashed curves) sections: 1 - AP-AP; 2 - AP-Binder; and 3 Binder-Binder

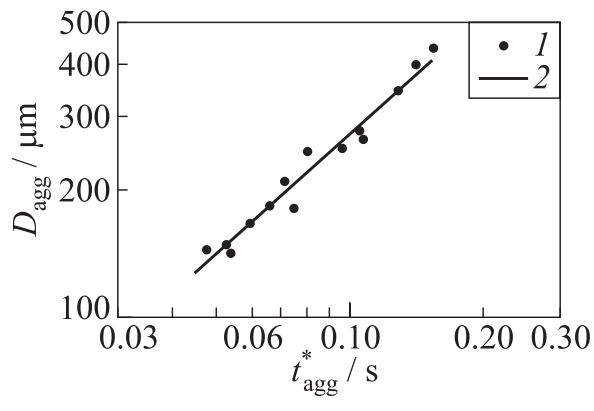

Figure 11 Correlation between characteristic time $\left(t_{\text {agg }}^{*}\right)$ and agglomerate size $\left(D_{\text {agg }}\right): 1$ - experimental; and $2-$ power fitting

The direct comparison of the TPPF obtained on experimental and numerical sections is reported in Fig. 10 and shows that the experimental method is not appropriate for the complete recognition of coarse AP on the test surface.

In fact, when $r \rightarrow 0$, the experimental volumetric fraction of AP is clearly underestimated, while the TPPF of numerically-generated sections is correct $(40 \%$ for the former and $55 \%$ for the latter). When the coarse AP distribution is considered, finer particles are more difficult to detect than the larger ones and this may explain the gap. Moreover, Two-Point Probability curves from the experimental sections feature smoothed shapes and the definition of a characteristic size becomes impossible. For this reason, the numerical generation of propellant sections is the most adequate.

The characteristic size defined by the numerical simulations allows computing a corresponding characteristic time $t_{\text {agg }}^{*}$, which represents the survival time of the microstructure because of the combustion advancement: it can be simply calculated as two times the characteristic size divided by the burning rate. The characteristic time can be seen as the time available for particles aggregation, and rises for larger characteristic size of the microstructure or a lower burning rate. This parameter has been shown to correctly fit the mean volumetric diameter of agglomerate populations for four different propellants, featured by both bi-modal and three-modal AP distribution (Fig. 11). Agglomeration data are obtained with high-speed and high-resolution imaging and the measurement techniques described above. It can be noted that the correlation between $D_{\text {agg }}$ and $t_{\text {agg }}^{*}$ of the propellant microstructure can be fitted by a power fitting $D_{\text {agg }}=2432 t_{\text {agg }}^{0.95}$, very close to a linear dependence $D_{\text {agg }} \approx 2432 t_{\mathrm{agg}}^{*}$. The adequacy of this relationship and its possible use for the prediction of agglomerate size based on starting formulation and burning rate is currently under test at SPLab. 


\section{DETAILED DESCRIPTION OF AGGLOMERATES FROM THREE-DIMENSIONAL STATISTICS}

The 3D statistics works for volumetric particle packs of spheres. Given the information regarding propellant composition, namely, material fractions and their mean size, a material representative is generated by arranging a mixture of particle families, each represented by single size spheres, in a 3D domain by means of a packing code. Although the use of a sphere as a representative of ingredients is a simplification, the actual shapes are similar being space-grade Al of spherical shape and AP of an ellipsoidal shape. One more simplification, adopted for a matter of reducing CPU time, applies to finer oxidizer components which are homogenized into the binder, provided that the homogenization does not involve a consistent volume fraction. In any case, the number of items to represent and pack per family should be statistically significant and indications from prior research showed that a minimum of one thousand particles is necessary to allow for convergence of the statistical descriptors. Following up this constraint, four propellant mockups were generated as listed in Table 1 as a replication of real compounds. Although the same raw materials are used in all compositions, two of them ( $A$ and $B$ ) come from a lab-scale facility and two $(C$ and $D)$ are from an industrial supplier. Marked differences in AP powder size can be appreciated: propellants $A$ and $D$ have a similar composition, propellant $C$ has a consistent fraction of $400 \mu \mathrm{m}$ AP while propellant $B$ has a single size oxidizer, without any fine component to homogenize into the binder.

The analysis of the packs follows simple criteria of proximity, laying on the assumption that the agglomeration is likely to involve neighboring metal particles. After a radius of collection is set at the beginning of the procedure, each particle is ideally surrounded by a spherical volume that discriminates whether metal spheres contribute to grow the same agglomerate or not (Fig. 12). In this sense, all particles within this basin of collection are meant to feed the same agglomerate. The statistical code, written in $\mathrm{C}++$ and parallelized through MPI, needs the computation of all sphere-to-sphere distances and the comparison to the threshold radius. At the end of the process, a list of CCP is produced, one per particle originally present in the pack. The generation follows the rules stated above, considering that one particle is not dropped as it enters one basin of collection but is still available to contribute to the basin generated by other

Table 1 Three-dimensional numerical propellant mockups

\begin{tabular}{|c|c|c|c|c|c|c|c|}
\hline \multirow{2}{*}{$\begin{array}{c}\text { Raw } \\
\text { material }\end{array}$} & \multirow{2}{*}{$\begin{array}{c}\text { Number } \\
\text { of particles }\end{array}$} & \multicolumn{4}{|c|}{ AP } & \multirow{2}{*}{$\mathrm{Al} 30 \mu \mathrm{m}$} & \multirow{2}{*}{ Binder } \\
\hline & & $400 \mu \mathrm{m}$ & $200 \mu \mathrm{m}$ & $150 \mu \mathrm{m}$ & fine & & \\
\hline $\bar{A}$ & 500,000 & $0 \%$ & $58 \%$ & $0 \%$ & $10 \%$ & $18 \%$ & $14 \%$ \\
\hline$B$ & 300,000 & $0 \%$ & $0 \%$ & $68 \%$ & $0 \%$ & $15 \%$ & $17 \%$ \\
\hline$C$ & 650,000 & $32 \%$ & $24 \%$ & $0 \%$ & $13 \%$ & $19 \%$ & $12 \%$ \\
\hline$D$ & 500,000 & $0 \%$ & $55 \%$ & $0 \%$ & $14 \%$ & $19 \%$ & $12 \%$ \\
\hline
\end{tabular}



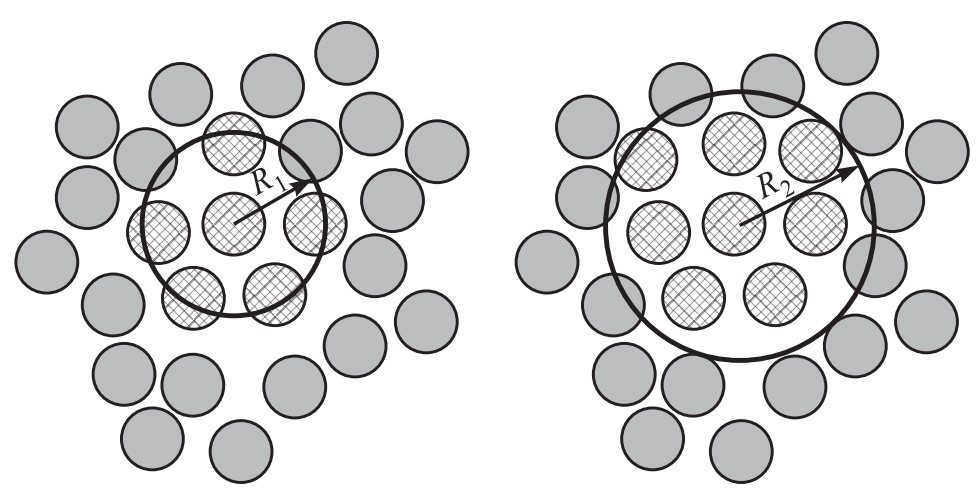

Figure 12 Threshold radius and collected metal particles for two examples

neighbors. This model relies only on geometric assumptions but retains a parameter of dependence (the threshold radius of collection) that may be used to accomplish other kinds of influence (for instance, pressure), once a formal connection is provided. As is, the parameter represents the number of effectively molten particles that merge together and may be connected to the residence time of metal particles on the burning surface. Currently, this technique does not operate a full prediction of the agglomeration process and some experimental information is requested for the choice of the collection radius. Further developments are currently ongoing in order to overcome this limitation.

Some parametric studies on the formulations of Table 1 are carried out. The primary interest is posed on the influence of the threshold radius on results and on the robustness of the algorithm, monitoring the variation of both mean statistical features and the detailed size distribution of the CCP generated by the analysis (Table 2 and Figs. 13 and 14). It is clear that the heterogeneity heavily affects the results of the model since agglomerates change size and distribution as

Table 2 Mean statistical features of the agglomerate size distributions generated by the model mockups

\begin{tabular}{|c|c|c|c|c|c|c|}
\hline $\begin{array}{c}\text { Curve } \\
\text { (Fig. 13) }\end{array}$ & $R, \mu \mathrm{m}$ & Mean, $\mu \mathrm{m}$ & $d[4,3], \mu \mathrm{m}$ & $R, \mu \mathrm{m}$ & Mean, $\mu \mathrm{m}$ & $d[4,3], \mu \mathrm{m}$ \\
\hline & \multicolumn{3}{|c|}{ (a) Propellant $A$} & \multicolumn{3}{|c|}{ (b) Propellant $B$} \\
\hline 1 & 117 & 127 & 133 & 79 & 82 & 86 \\
\hline 2 & 136 & 145 & 151 & 96 & 95 & 100 \\
\hline \multirow[t]{2}{*}{3} & 156 & 163 & 170 & 114 & 110 & 114 \\
\hline & \multicolumn{3}{|c|}{ (c) Propellant $C$} & \multicolumn{3}{|c|}{ (d) Propellant $D$} \\
\hline 1 & 166 & 185 & 193 & 124 & 137 & 143 \\
\hline 2 & 208 & 228 & 236 & 142 & 155 & 162 \\
\hline 3 & 249 & 274 & 282 & 162 & 174 & 181 \\
\hline
\end{tabular}




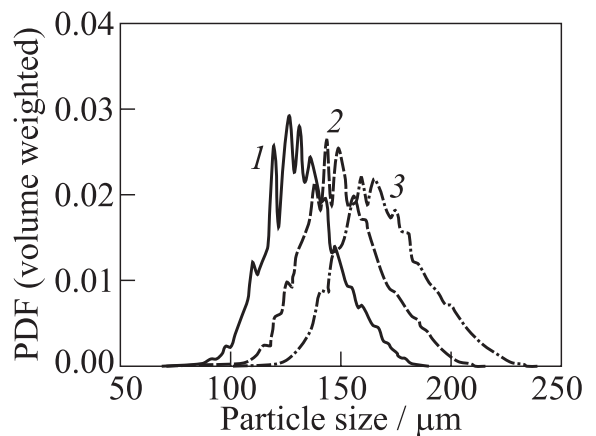

(a)

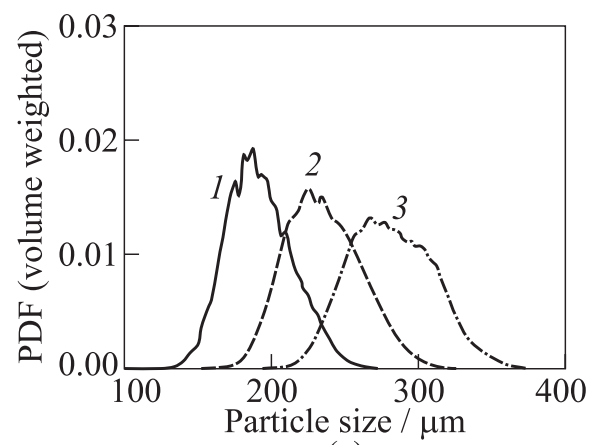

(c)

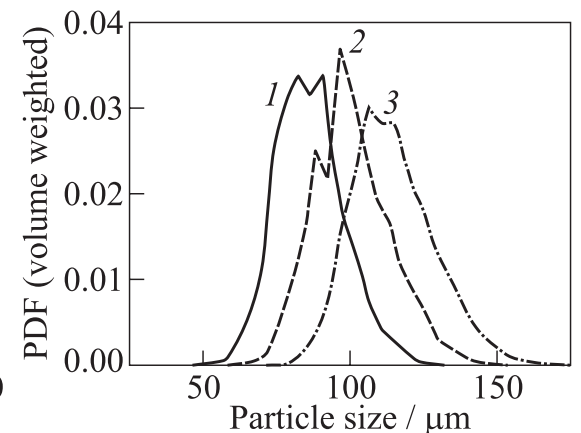

(b)

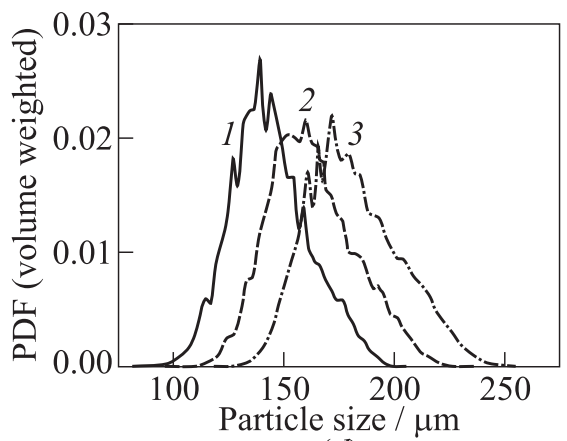

(d)

Figure 13 General model tests: PDFs of agglomerates

the threshold radius of collection is varied. The algorithm is robust over a wide range of the threshold radius supplying results of real-like CCP distributions but it does not include a combustion model. It follows that the agglomerate size cannot be lower than the smallest diameter already present in the pack.

At this standpoint experimental datasets of propellant agglomerates are contrasted to the size distributions generated by the model. Taking advantage of the nonintrusive measurement performed by means of high-speed movies, through manual and automated techniques described in section 3, combustion tests for propellants of Table 1 were postprocessed and the mean features of the populations are listed in Table 3.

A couple of sample cases are selected from both experiments and model data, reporting the relevant PDFs. The mass-weighted mean diameters $(d[4,3])$ of Tables 2 and 3 are used to correlate the model case to experimental combustion pressure.

The matching with experimental data looks considerably good and confirms that the geometric approach based on heterogeneity is adequate with this class of composite propellants. The interval and the maximum of the curves are well rep- 


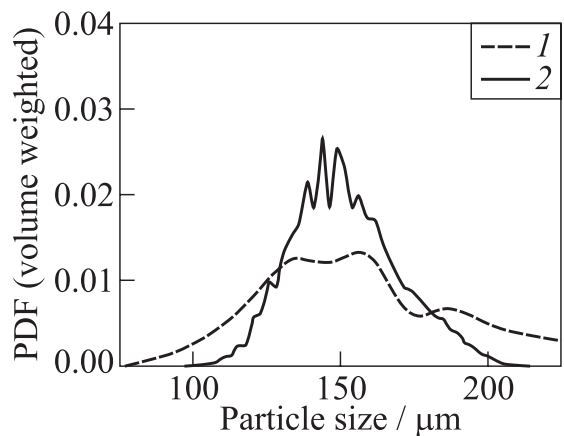

(a)

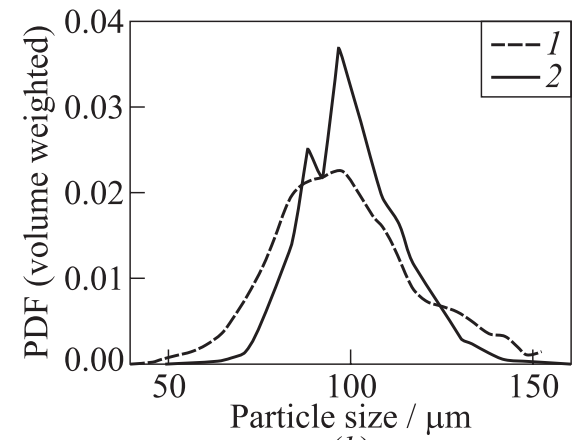

(b)

Figure 14 Comparison of experimental (1) and model (2) agglomerate PDFs for specific cases: (a) propellant $A, p=20$ bar, $R=136 \mu \mathrm{m}$; and (b) propellant $B$, $p=10$ bar, $R=96 \mu \mathrm{m}$

Table 3 Agglomeration data from experimental combustion tests

\begin{tabular}{rcc|rcc}
\hline$p$, bar & Mean, $\mu \mathrm{m}$ & $d[4,3], \mu \mathrm{m}$ & $p$, bar & Mean, $\mu \mathrm{m}$ & $d[4,3], \mu \mathrm{m}$ \\
\hline \multicolumn{3}{c|}{$(a)$ Propellant $A$} & \multicolumn{3}{c}{$(b)$ Propellant $B$} \\
\hline 5 & 175 & 192 & 5 & 98 & 114 \\
10 & 154 & 175 & 10 & 87 & 98 \\
20 & 136 & 155 & 20 & 67 & 78 \\
25 & 129 & 146 & 25 & N.A. & N.A. \\
\hline \multicolumn{3}{c}{ (c) } & Propellant $C$ & \multicolumn{3}{c}{$(d)$ Propellant $D$} \\
\hline 5 & 216 & 346 & 5 & 175 & 254 \\
10 & 156 & 248 & 10 & 120 & 181 \\
20 & N.A. & N.A. & 20 & 82 & 151 \\
25 & 96 & 177 & 25 & 75 & 141 \\
\hline
\end{tabular}

resented as well as for the range of CCP. Based on these experimental data, the model overestimates the central part of the distribution, while a lower smoothing feature is observed on the sides of the curve. Especially for propellant $B$, where experimental data of higher quality are available, the model comes closer to the real data, reproducing quite well the steepness of the central peak. On the other hand, the algorithm is capable of producing only monomodal populations, as visible from the general test run in the previous pages, and captures only the main peak of agglomerate size, dropping the smaller fractions.

\section{CONCLUDING REMARKS}

This work addressed propellant metal particle agglomeration from experimental and modeling viewpoints, aiming at the description of some modern methods to 
deal with characterization, interpretation, and size prediction of $\mathrm{CCP}$ as they are released from the burning surface. In fact, as CCP lift from the burning surface, they are not yet subjected to the environmental constraints that modify size and composition. This data can be useful not only to foster a better understanding of metal combustion inside rocket chambers but also as input for multiphase core flow simulations.

In the first part of the work, the implementation and the results of an automated postprocessing tool specific for agglomeration measurement were described. The software was an extension of an application available under public domain license and was applied to process high-speed and high-resolution imaging acquisitions. Automated and consolidated manual techniques were compared showing an agreement between the results of the two methods. Larger number of particle measurements for a limited time and operator-independence of results were the advantages of the new technique, while higher sensitivity to imaging quality and limited range of application for the combustion pressure were the drawbacks.

The modeling section took advantage of recent developments in packing codes and applied simple statistical tools to characterize heterogeneity. The approaches were grounded on the pocket concept, formerly introduced by Cohen, assuming that agglomeration is favored inside the fuel-rich regions naturally present in composite solid propellants. Two- and three-dimensional methodologies were presented for fitting experimental data as well as for the detailed description of agglomerate population, once a mean feature is given. The comparison with experimental results demonstrated that the statistical treatment of agglomeration could capture main trends showed by the propellants tested. Yet, in order to have a fully comprehensive tool, statistics and geometric criteria should be enriched with CCP combustion models. Moreover, the current approach alone cannot predict agglomerates from the sole heterogeneous structure. Current developments include a criterium to make the model dependent on ignition features of the agglomerates generated at/near the propellant surface.

\section{ACKNOWLEDGMENTS}

Three-dimensional packing code in use is the property of CSAR, University of Illinois at Urbana-Champaign. Supercomputing resources and MPI environments were granted by CINECA and CILEA.

\section{REFERENCES}

1. Hermsen, R. W. 1981. Aluminum oxide particle size for solid rocket motor performance prediction. J. Spacecraft Rockets 18(6):483-90.

2. Reydellet, D. 1986. Performance of rocket motors with metallized propellants. AGARD AR-230. 
3. Babuk, V.A., V.A. Vasilyev, and M. S. Malakhov. 1999. Condensed combustion products at the burning surface of aluminized solid propellant. J. Propul. Power 15(6):783-93.

4. Babuk, V.A. 2007. Problems in studying formation of smoke oxide particles in combustion of aluminized solid propellants. Combust. Explosion Shock Waves 43(1):3845.

5. Beckstead, M.W. 2002. A summary of aluminum combustion. RTO-EN 023, NATO.

6. Babuk, V. A., V. A. Vassiliev, and V. V. Sviridov. 2001. Propellant formulation factors and metal agglomeration in combustion of aluminized solid rocket propellants. Combust. Sci. Technol. 163:261-89.

7. Abramoff, M.D., P. J. Magelhaes, and S. J. Ram. 2004. Image processing with ImageJ. Biophotonics Intern. 11(7):36-42.

8. Soille, P., and L. Vincent. 1991. Watersheds in digital spaces: An efficient algorithm based on immersion simulation. IEEE Trans. Pattern Anal. Machine Intelligence 13(6):583-98.

9. Serra, J. 1982. Image analysis and mathematical morphology. London: Academic Press.

10. Povinelli, L. A., and A. Rosenstein. 1964. Alumina size distributions for highpressure composite solid-propellant combustion. AIAA J. 2(10):1754-60.

11. Cohen, N. S. 1983. A pocket model for aluminum agglomeration in composite propellants. AIAA J. 21(5):720-25.

12. Babuk, V.A., V.A. Vasil'ev, and V.V. Sviridov. 1999. Modeling the structure of composite solid rocket fuel. Combust. Explosion Shock Waves 35(2):35-40.

13. Rashkovskii, S. A. 2002. Role of the structure of heterogeneous condensed mixtures in the formation of agglomerates. Combust. Explosion Shock Waves 38(4):435-45.

14. Maggi, F. 2007. Center for simulation of advanced rockets. University of Illinois at Urbana Champaign. Internal Report. Urbana, IL, USA.

15. Collins, B., F. Maggi, K. Matous, T. Jackson, and J. Buckmaster. 2008. Using tomography to characterize heterogeneous propellants. AIAA Paper No. 2008-941.

16. Gallier, S., and F. Hiernard. 2008. Microstructure of composite propellants using simulated packings and X-ray tomography. J. Propul. Power 24:147-50.

17. Bandera, A., F. Maggi, and L. DeLuca. 2009. Agglomeration of aluminized solid rocket propellants. AIAA Paper No. 2009-5439.

18. Maggi, F., A. Bandera, L. Galfetti, L. T. De Luca, and T. L. Jackson. 2010. Efficient solid rocket propulsion for access to space. Acta Astronautica 66(11-12):1563-73. doi:10.1016/j.actaastro.2009.10.012.

19. Gallier, S. 2011. Heterogeneous solid propellants: From microstructure to macroscale properties. In: Progress in propulsion physics. Eds. L. DeLuca, C. Bonnal, O. Haidn, and S. Frolov. EUCASS advances in aerospace sciences book ser. TORUS PRESS, EDP Sciences. 2:21-34.

20. Bandera, A. 2009. Combustion of metallized solid rocket propellants and motor performance. Ph.D. Thesis in Energetics, Politecnico di Milano. Milan, Italy.

21. Stafford, S., F. Maggi, T. Jackson, and J. Buckmaster. 2008. Propellant packs. AIAA Paper No. 2008-937.

22. Maggi, F., S. Stafford, T. L. Jackson, and J. Buckmaster. 2008. Nature of packs used in propellant modeling. Phys. Rev. E 77(046107):1-17. doi:10.1103/ PhysRevE.77.046107.

23. Stoyan, D., and H. Stoyan. 1994. Fractals, random shapes and point fields: Methods of geometrical statistics. Wiley. 